\title{
EXPERIMENTAL AND NUMERICAL STUDY OF LOW ASPECT RATIO ASYMMETRIC DIFFUSER
}

W. A. Aissa ${ }^{1}$, M. S. Eissa ${ }^{2}$,

W. A. Abdel-Fadeel ${ }^{2}$ and I. K. Mohamed ${ }^{2}$

${ }^{1}$ Corresponding author: Associate Professor, Mechanical Power Department, High Institute of Energy, South Valley University, Aswan, Egypt, email:walidaniss@yahoo.com,

${ }^{2}$ Assistant Professor, Mechanical Power Department, High Institute of Energy, South Valley University, Aswan, Egypt

Tel/Fax: + [20] 973480450

(Received February24, 2009 Accepted March 11, 2009)

The goal of the present study is to investigate the flow through an asymmetric low aspect ratio planar diffuser. The flow which is fully developed channel flow inlet conditions is both experimentally investigated and computed using commercial cfd code, fluent. Rng $k-\varepsilon$ model is used as a turbulence model and standard wall function is used. The questions about the two-dimensionality of the flow are investigated. The Reynolds number of diffuser inlet flow is $3.5 \times 105$ based on the centerline velocity and the channel height. Flow is visualized to get insight view of the flow map in the diffuser.

KEYWORDS: Asymmetric diffuser, fluent code, rng $k-\varepsilon$ model, flow visualization, experimental

\section{NOMENCLATURE}

\begin{tabular}{|lllll|}
\hline $\mathrm{H}$ & $\begin{array}{l}\text { Asymmetric diffuser } \\
\text { channel height }\end{array}$ & & \\
$\mathrm{K}$ & $\begin{array}{l}\text { Turbulence kinetic energy } \\
\mathrm{u}, \mathrm{v},\end{array}$ & $\begin{array}{l}\text { Components of } \mathrm{V} \text { in } \mathrm{x}, \mathrm{y}, \mathrm{z} \\
\mathrm{E}\end{array}$ & Rate of dissipation of $\mathrm{k}$ \\
$\mathrm{w}$ & directions & & & \\
$\mathrm{x}, \mathrm{y}$, & Cartesian coordinates directions & $\theta$ & Diffuser angle \\
$\mathrm{z}$ & & & \\
$\mathrm{V}$ & Velocity vector & & \\
\hline
\end{tabular}

\section{INTRODUCTION}

Traditional diffuser is known as ax-symmetric diffuser, it is homogenous around its axis. Diffusers may be found in irregular shape for special applications, in this case it is known as asymmetric diffuser. Therefore, the term asymmetric indicates that the diffuser is not homogenous around its axis.

It is used in many applications in energy technology such as the draft tube in hydropower plants, gas and steam turbines, air conditional ducts. Separation in these flows may drastically reduce the efficiency of the device or even jeopardize its 
function. New insights in the area of separation, separation control may therefore have large energy and environmental impact because of improved efficiencies, reduced fuel consumption and exhaust. Better models for separation prediction may also improve flow quality and lower development costs in a variety of industrial applications. Prediction of separation is therefore crucial. Today this is done largely with numerical methods. However simulations of complex turbulent flows with separation are still subject to large quantitative errors and to improve the models it is necessary to have access to high quality data from wall defined geometries and with carefully specified inlet conditions.

In general, diffuser is a gradual enlargement device constructed to decelerate a high-velocity fluid in a manner that results in an increase in the pressure of that fluid. In essence, its function is the exact opposite of the nozzle.

Simple diffusers have been extensively studied and are well understood. In fact, they are presently a standard topic covered in introductory fluid mechanics textbooks. One great limitation of simple diffusers is their inability to spread the flow much more than 7-degrees. Beyond this, separation occurs within the diffuser, effecting increased losses and thus decreased efficiency in terms of pressure recovery. If a much higher degree of spreading is desired, an advanced diffuser design is required.

Asymmetric diffuser was both experimentally and numerically investigated. Buice and Eaton [1], Mathes [2] and Feakins et al. [3] conducted experimental work, while other investigators Gravemeier [4]-Schlüter et al. [12], numerically treated flow within asymmetric diffuser.

Buice and Eaton [1] provided measurements for a two-dimensional diffuser flow which includes separation and reattachment forming a separation bubble on one wall of the diffuser. They stated that Separated flows are difficult to predict because the separating and reattaching boundary layers are highly out of equilibrium and that advanced simulation techniques including large eddy simulation and detached eddy simulation were being developed to compute separated flows. In addition they stated that, such simulations require realistic, time-dependent in-flow conditions and that these are most easily generated if the inlet flow is simple.

Mathes [2] devised and performed experimental analysis of airflow through an advanced-design, quasi-radial diffuser receiving an oscillating jet induced by a rotating baffle. The initially unsteady flow was found to lose its unsteadiness as it progressed through the diffuser and exited into the atmosphere.

Feakins et al. [3] experimentally investigated the effect of a varying downstream boundary condition on dynamic separation control in a two-dimensional low-speed asymmetric diffuser. They stated that the potential for coupling between the downstream boundary condition and the separation dynamics is relevant, for example, in using separation control to enable more aggressive serpentine aircraft inlets, where the compressor may be close to the separation point.

Gravemeier [4] applied the variational multiscale Large Eddy Simulation (LES) to turbulent flow in a planar asymmetric diffuser. The Reynolds number; based on the half-width of the inlet channel and the streamwise bulk mean velocity of the investigated flow, was about 10,000. Gravemeier stated that several features of turbulent flow in a diffuser indicate its higher complexity such as large unsteady separation bubbles, change in the streamwise pressure gradient and slowly growing 
internal layer that emerges at the upper wall in the relaxation zone downstream of the sharp variation in the streamwise pressure gradient.

Sbrizzai and Soldati [5] studied the behavior of inertial particles inside a curved-profile diffuser using an Eulerian-Lagrangian approach. The three-dimensional fully developed turbulent flow field was simulated using LES and exploiting the immersed boundaries approach to model the shape of the computational domain. The Reynolds number of the flow in the inlet pipe based on bulk velocity was 17560 . They observed that the main coherent structures, i.e. vortex rings, are the same. In addition they stated that they observed secondary vortex rings, but these seem to have no role in particle dispersion.

The aim of Törnblom et al. [6] was to investigate ways to reduce the size and/or the motion of the separated region in plane asymmetric diffuser. They stated that vortex generators introduce streamwise vortices which bring fluid with a high level of streamwise momentum close to the wall. They stated that their control strategy was to introduce spanwise vortices in the flow to break its spanwise coherence. In addition, they stated that vortex generators are efficient when it comes to delay separation.

The goal of the study presented by Kaltenbach [7] was to investigate the diffuser flow to see if the wall-model based LES method can be applied. The statistics from a well resolved LES of the diffuser flow were used to study the near-wall zone in order to see (i) what are the relevant terms in the mean momentum balance and (ii) whether the turbulent shear stress in the near-wall layer can be predicted by an algebraic eddy-viscosity model.

Dahlström and Davidson [8] used a hybrid RANS/LES method to solve a one-equation turbulence model in the region from the wall and out. Computations had been conducted on an asymmetric diffuser, using a mesh with insufficient LES resolution in the near-wall region. They stated that in order to increase the number of tests, the majority of the computations had been conducted on just the inlet channel of the diffuser.

LES of the turbulent flow in an asymmetric planar diffuser was carried out as shown in the work by Suh,[9] using FLUENT. The inlet conditions were specified as a fully-developed channel flow at $\mathrm{Re}=20,000$ based on the centerline velocity and the channel height. It was stated that the result from unsteady LES can show large recirculating zones and non-equilibrium due to the adverse pressure gradient in the diffuser, which can't be predicted by RNG k- $\varepsilon$ model. However, it also predicts spurious recirculating region, which is unphysical. It was found that accurate representation of the inflow velocity field and outflow condition is critical for accurate prediction of flow field in the diffuser

Results from a computational study of the separated flow behavior through a 2-D asymmetric subsonic diffuser computed with the Wind CFD code had been presented as shown in the work by DalBello et al. [10]. Using the SST turbulence model, a grid sensitivity study was performed by varying the number of grid points in the transverse direction across the diffuser. A sensitivity study of the initial grid point spacing was also performed.

Iaccarino [11] reported a comparison between three CFD commercial codes for the turbulent flow in a planar asymmetric diffuser. Two turbulence models were used: the first was the Low-Reynolds $k-\varepsilon$ model (with Launder and Sharma damping functions) which is available as a standard feature in the codes; the second was the $v 2$ - 
$f$ model, which was implemented through user defined routines. The same grid and the same spatial discretization were used for all the simulations.

Schlüter et al. [12] reported an internal layer found in the turbulent flow through an asymmetric planar diffuser using large-eddy simulation. They discussed five issues relevant to the internal layer: definition and identification, conditions for occurrence, connection with its outer flow, similarity with other equilibrium flows, and growth.

The objective of the present work is to conduct experimental and numerical study of asymmetric diffuser which configuration is analogues to that of the diffuser geometry shown in Fig.1 replicates the geometry of Obi et al. described by Buice and Eaton [1]. However, the aspect ratio is lower to study its effect on the two-dimensionality of the flow.

Numerous tests of the two-dimensionality are made in the current work through measurements of the spanwise uniformity of the mean velocity. The tests show that the flow is two dimensional within the measurement resolution.

\section{EXPERIMENTAL TEST SETUP}

\subsection{Introduction}

The experiments are conducted in the blower-driven low speed wind tunnel of High Institute of Energy modified with an additional attachment of plane wall asymmetric diffuser, with a channel height; $\mathrm{H}$ of $64 \mathrm{~mm}$ and a width of $375 \mathrm{~mm}$. The $(5.86: 1)$ aspect ratio inlet channel is $1.0 \mathrm{~m}$ long providing fully developed channel flow at the inlet of the diffuser test section. The diverging angle of the diffuser is $7^{\circ}$, while the downstream section height is $4.7 \mathrm{H}$ followed by constant height extension section. The diffuser geometry is illustrated in Fig.1. Flow Reynolds number, based on inlet velocity and inlet section height is $3.5 \times 10^{5}$. A Perspex material with a thickness of 5 $\mathrm{mm}$ is used for constructing the diffuser body for the purpose of flow visualization.

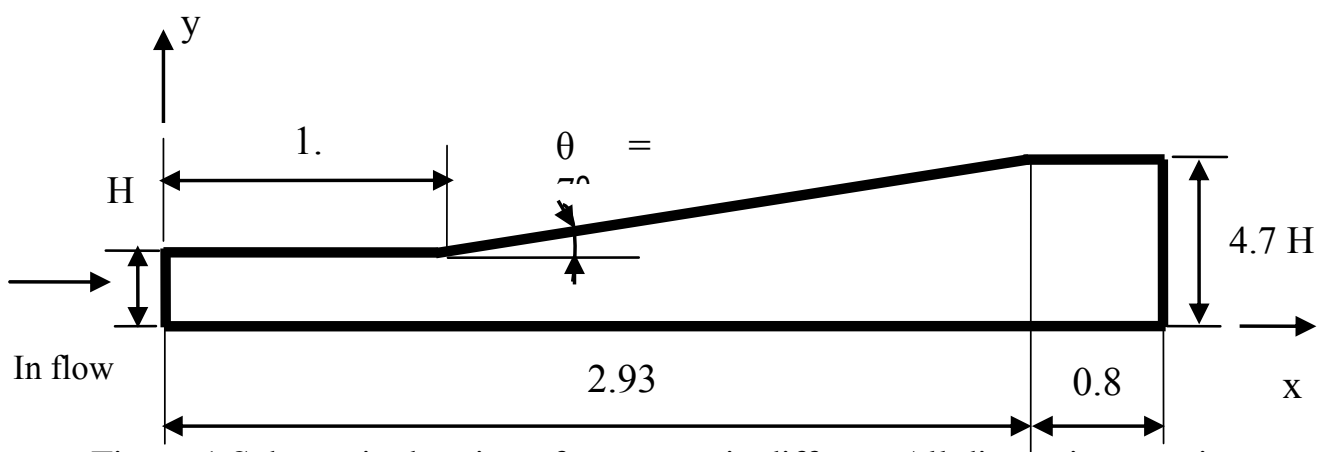

Figure 1 Schematic drawing of asymmetric diffuser. All dimensions are in $\mathrm{m}$

\subsection{MEASURING TECHNIQUE AND MEASURING POINTS}

Velocity profile survey is performed using a calibrated five hole probe. The measuring points are situated at the base sheet of the asymmetric diffuser and its extension; as illustrated in Fig. 2. The probe is moved vertically to measure the velocity at five points dividing the height at each location equally. 


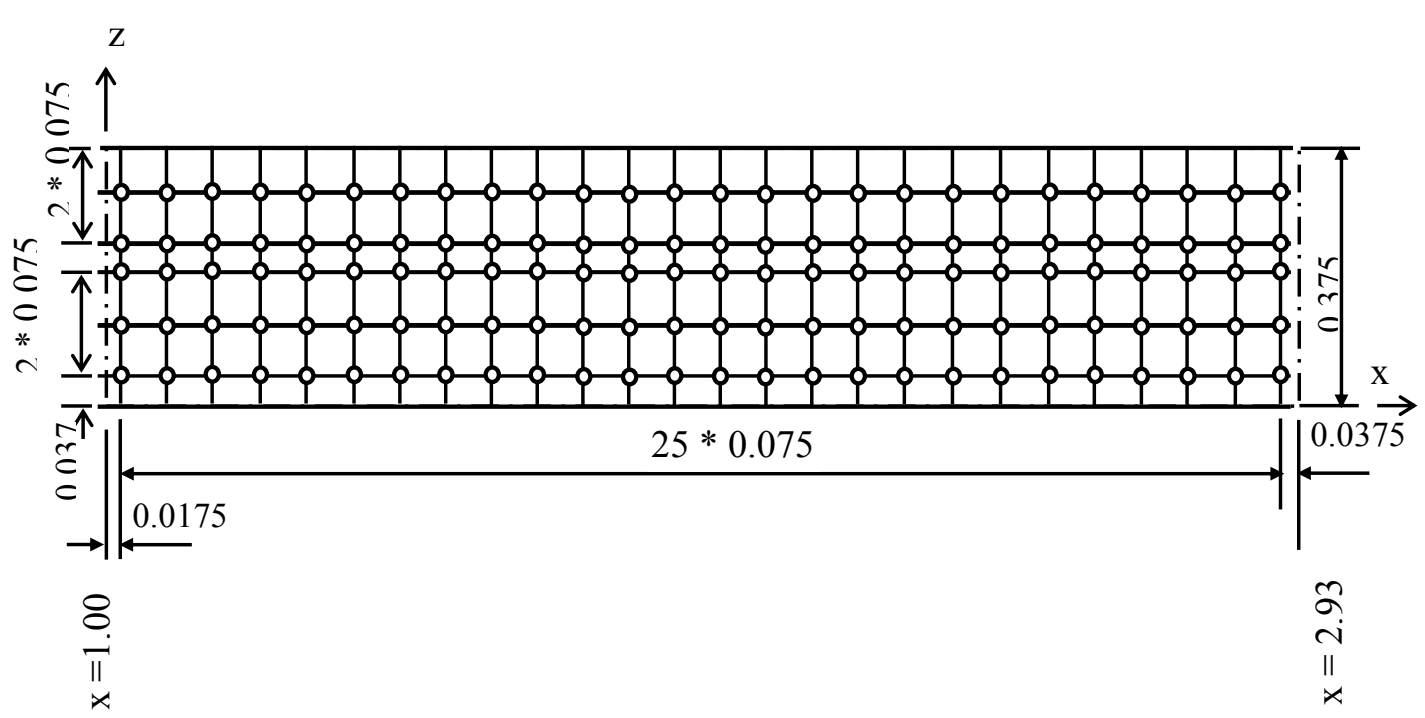

Figure 2 Top view illustrating positions of measuring points at the base of the diffuser; all dimensions are in $\mathrm{m}$ (entrance section is not included).

\section{FLOW VISUALIZATION}

The objective of this experiment is to visualize the flow stream lines during passing inward the diffuser. In addition, flow visualization illustrates the location of separation (if any). The separation line near the boundary layer for the upper diffuser surface is of primary interest for the asymmetric geometry. The experiments also give an understanding and indication of the surface streamlines and any weak vortices.

Flow visualization for the configurations under study using smoke generated from the burning of incense at the inlet section of the diffuser. This method implies that the smoke particles are sufficiently small mass so that they are carried freely at the flow velocity. The smoke particle technique can be considered a bit of an art when it comes to capturing and visualizing flow features that require significant time develop, such as the smoke-accumulation line that indicates separation. The quality of the captured photos was somewhat identical, and consequently a weak light is applied during the experiments. The inner surfaces are optimally cleaned to allow the smoke particles to move more easily during the tunnel run.

\section{NUMERICAL TECHNIQUE}

The flow is solved as $3 \mathrm{D}$, steady and incompressible. RNG k- $\varepsilon$ model as a turbulence model and standard wall function are used. The computations employ a hexahedral grid displaying 47926 nodes, 118884 faces and 35529 cells.

The boundary conditions are specified at all boundaries of solution domain since the set of governing equations are Elliptic P.D.E.s. At the inlet boundary, the boundary values for the inlet flow velocity, inlet temperature, inlet turbulence intensity and length scale are specified. At the outlet boundary, the value of outlet pressure is 
specified; whereas gradient conditions are used for turbulence intensity, length scale and temperature.

Solid wall boundary conditions are used for the diffuser model. Wall functions are used at all solid boundaries.

Here $\mathrm{X}, \mathrm{Y}$ and $\mathrm{Z}$ refer to display plane distances in the $\mathrm{x}, \mathrm{y}$, and $\mathrm{z}$ Cartesian coordinates directions, respectively; measured from an origin lying at the intersection of the bottom plane of the diffuser, the diffuser longitudinal plane, and the inlet channel plane. The $\mathrm{x}$-direction is aligned with the longitudinal axis of the model (also free stream direction), the y-direction with the vertical, and the $z$-direction with the horizontal lateral direction (also, horizontal cross-stream direction). It is remarked that, for reasons of clarity, the coordinate scales in the horizontal and vertical directions are very different which causes the diffuser shape to appear in unreal aspect ratio shape.

The grid system for whole domain and detail of the computational grid in the region close to the connection between the channel and the diffuser are shown in the Fig. 3.
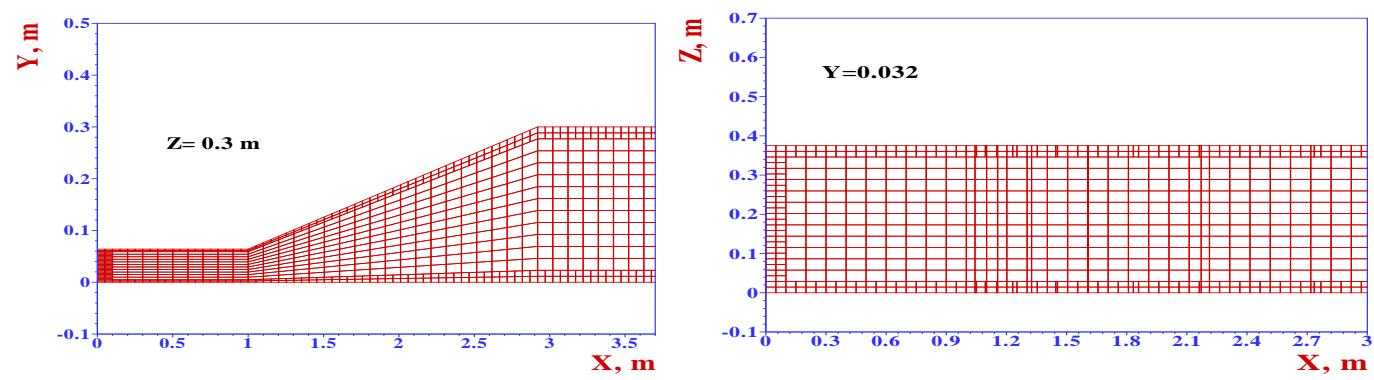

Figure 3 Asymmetric diffuser $X-Y($ at $Z=0.3 \mathrm{~m}$ ) and $X-Z$ (at $Y=0.032 \mathrm{~m}$ ) planes grid mesh.

\section{RESULTS AND DISCUSSION}

The flow in asymmetric, three dimensional, planar diffuser is considered. The inlet conditions are specified as a fully-developed channel flow at $\mathrm{Re}=3.5 \times 10^{5}$ based on the centerline velocity of $80 \mathrm{~m} / \mathrm{s}$; which is the same as measured value, and the channel height.

Figure 4 illustrates streamwise velocity vectors distribution through the longitudinal plane at $\mathrm{Z}=0.037 \mathrm{~m}$, computed with RNG k- $\varepsilon$ steady model. In which, the separation zone near the upper surface is shown. This result, disagree with Suh [9] who confirmed that the RNG k- $\varepsilon$ model can't predict separation in this flow field in a diffuser. This may be due to the difference in Reynolds number. Moreover, it was stated by Suh that Even though results from LES show better prediction than RNG k- $\varepsilon$ case, however their results are also very different from experimental results. DalBello et al. [10] stated that the RNG k- $\varepsilon$ model underpredicts the flow velocities in the core regions, slightly overpredicts the flow velocities towards the lower wall, and predicts a more uniform spread of velocities in the vertical direction in the core region. There is no separation region near the lower wall and the flow remains attached on it as may be seen in visualization scheme presented below. Fig. 4 predicts separation near the upper wall starting at $\mathrm{x}=1.5 \mathrm{~m}$. However, flow visualization shows separated flow 
downstream this streamwise location. In addition, it may be remarked that fast recovery to equilibrium flow field occurs downstream the separation zone.

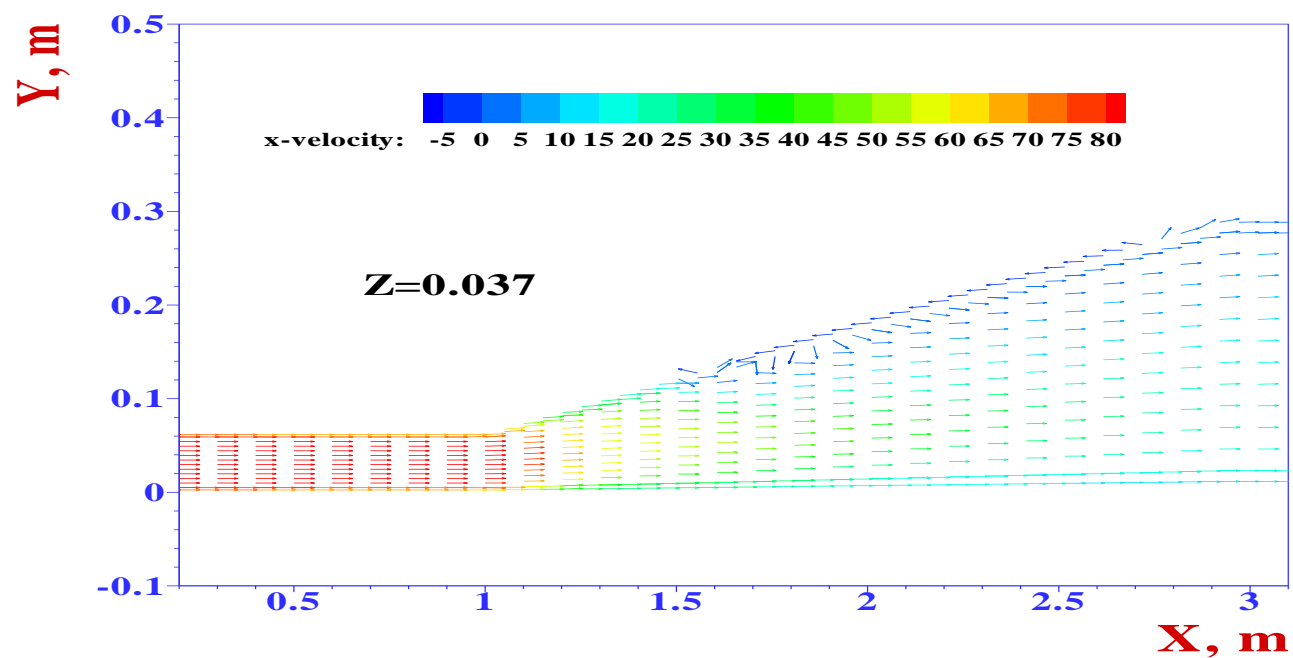

Figure $4 \mathrm{u}$ Velocity vectors distribution in $\mathrm{X}-\mathrm{Y}$ plane at $\mathrm{Z}=0.037 \mathrm{~m}$.

Figure 5 shows the stream-wise velocity distribution by RNG k- $\varepsilon$ turbulence model through the longitudinal plane at $\mathrm{Z}=0.187 \mathrm{~m}$. The figure shows the extent of the separation region. Relatively thickboundary layers can be seen in conjunction with a higher-speed core indicative of fully-developed channel flow. The flow enters the diffuser and separates on the upper wall at about $1.5 \mathrm{~m}$ due to the adverse pressure gradient created by the reduction in flow velocity.

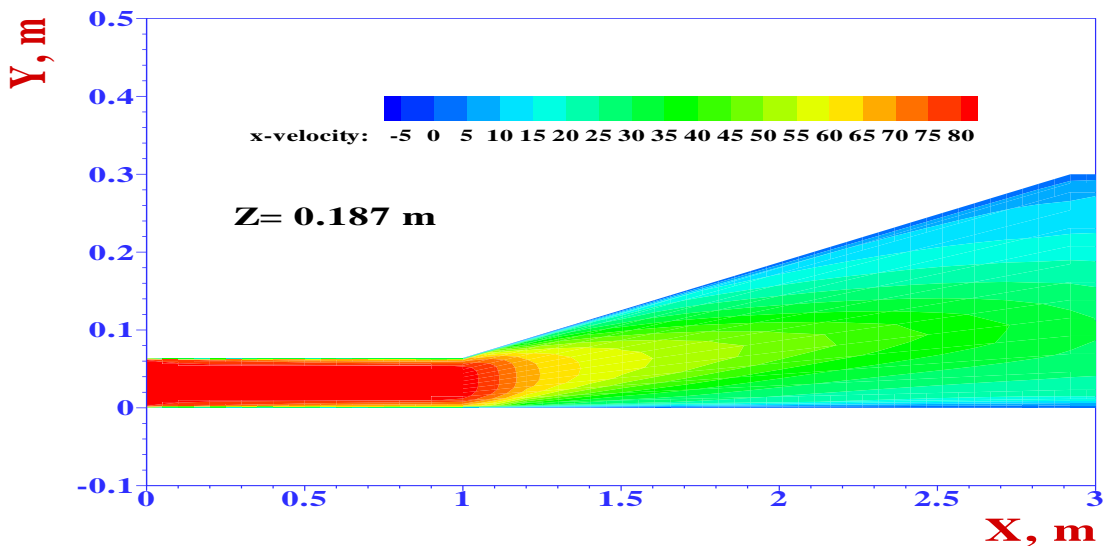

Figure 5 Streamwise $(\mathrm{u})$ velocity contours in $\mathrm{X}-\mathrm{Y}$ plane at $\mathrm{Z}=0.187 \mathrm{~m}$

The flow visualization for both the inlet channel and the diffuser and detail of the diffuser are shown in the Fig. 6. 

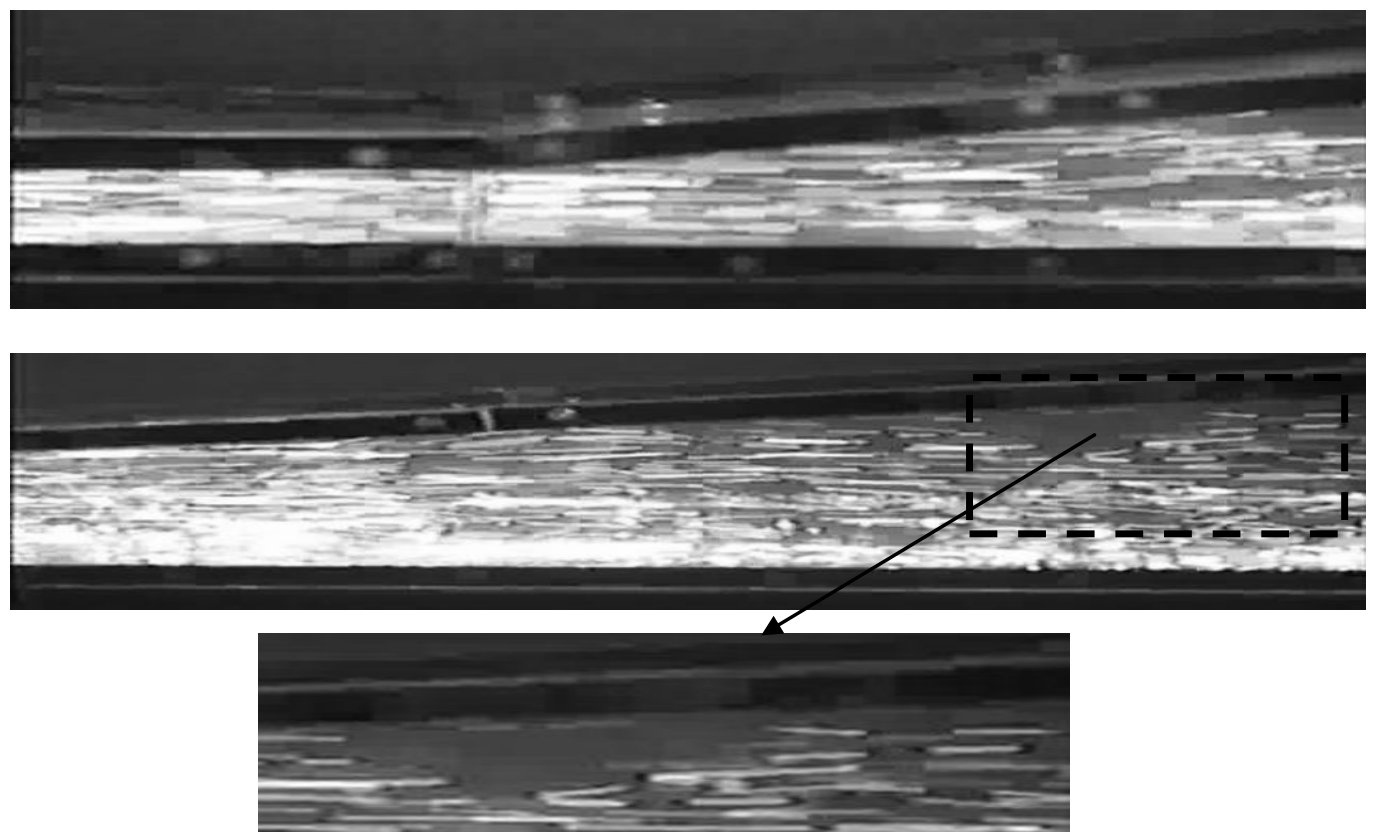

Figure 6 Asymmetric diffuser flow visualization.
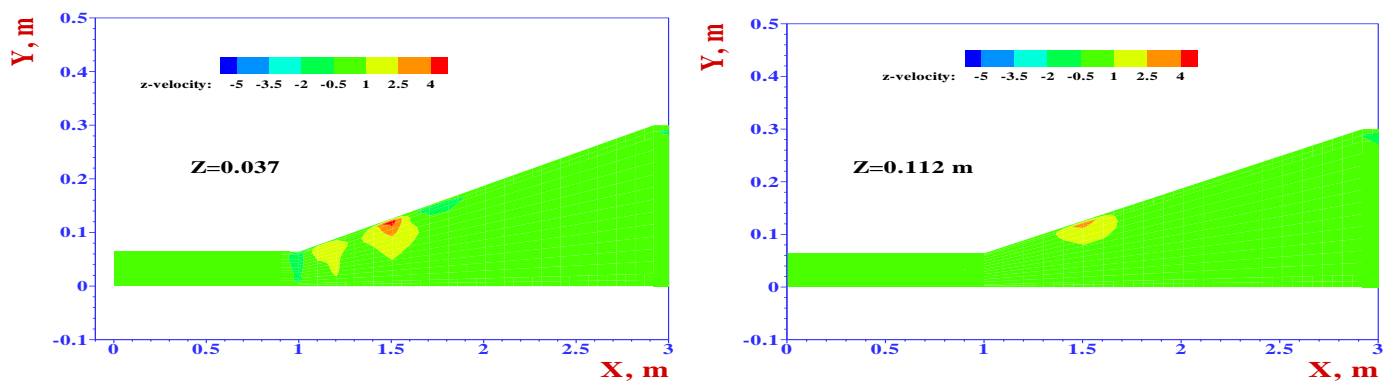

Figure $7 \mathrm{w}$ velocity contours in $\mathrm{X}-\mathrm{Y}$ plane at different $\mathrm{Z}$ values.
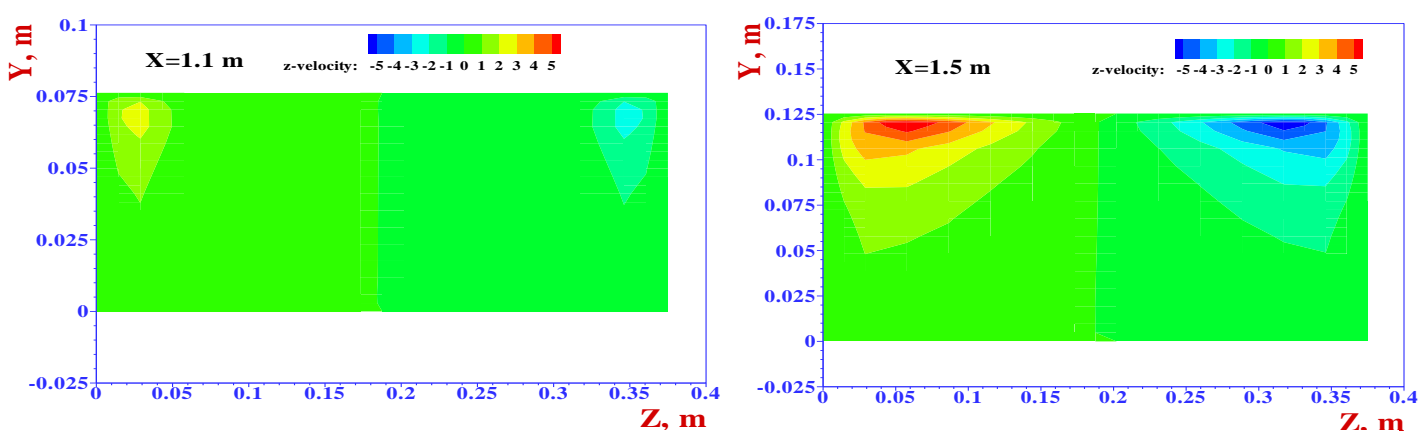

Figure $8 \mathrm{w}$ velocity contours in Z-Y plane at different $\mathrm{X}$ values. 

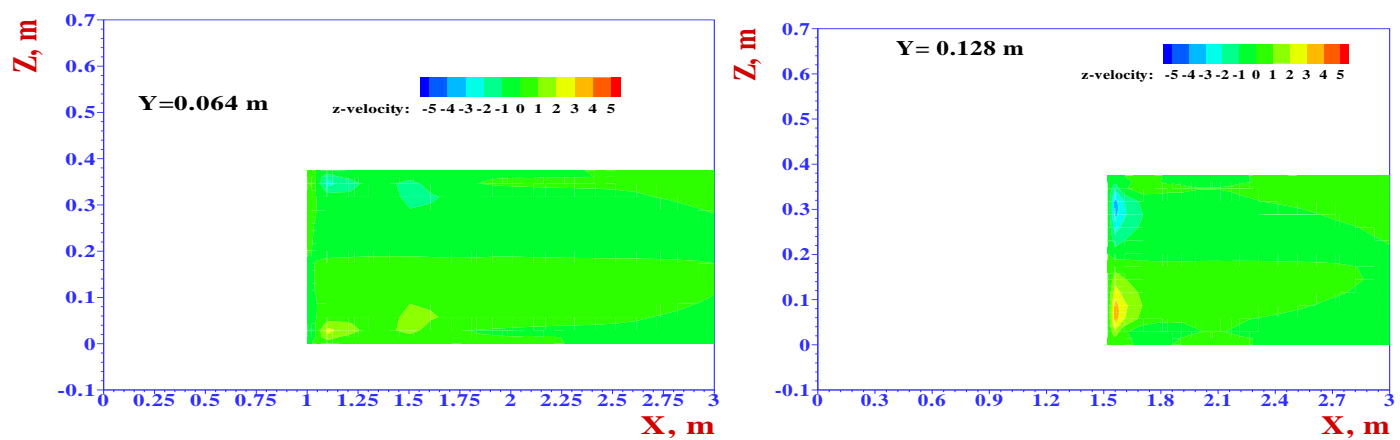

Figure $9 \mathrm{w}$ velocity contours in $\mathrm{X}-\mathrm{Z}$ plane at different $\mathrm{Y}$ values.
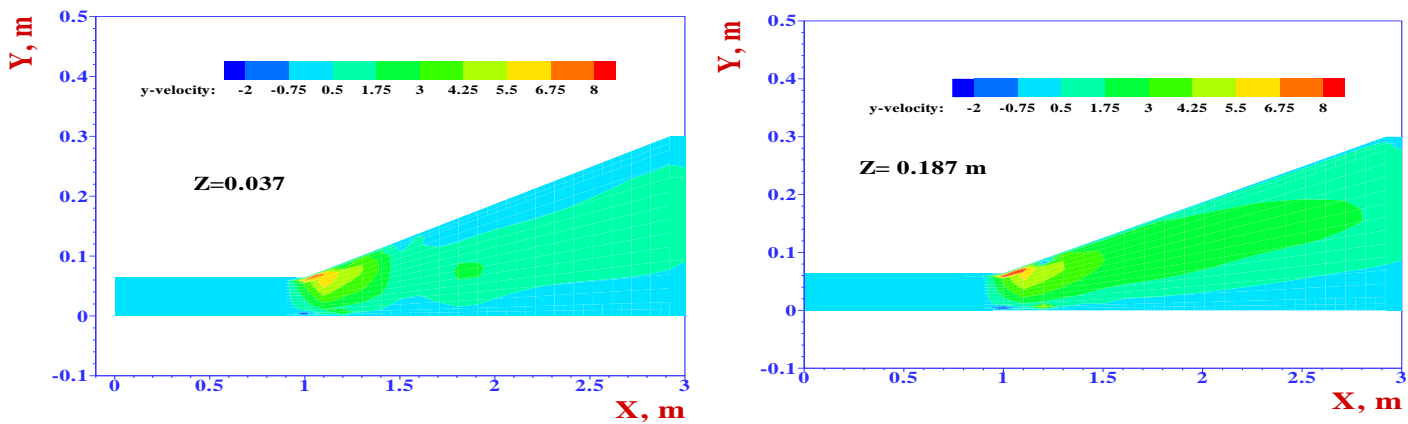

Figure $10 \mathrm{v}$ velocity contours in $\mathrm{X}-\mathrm{Y}$ plane at different $\mathrm{Z}$ values.
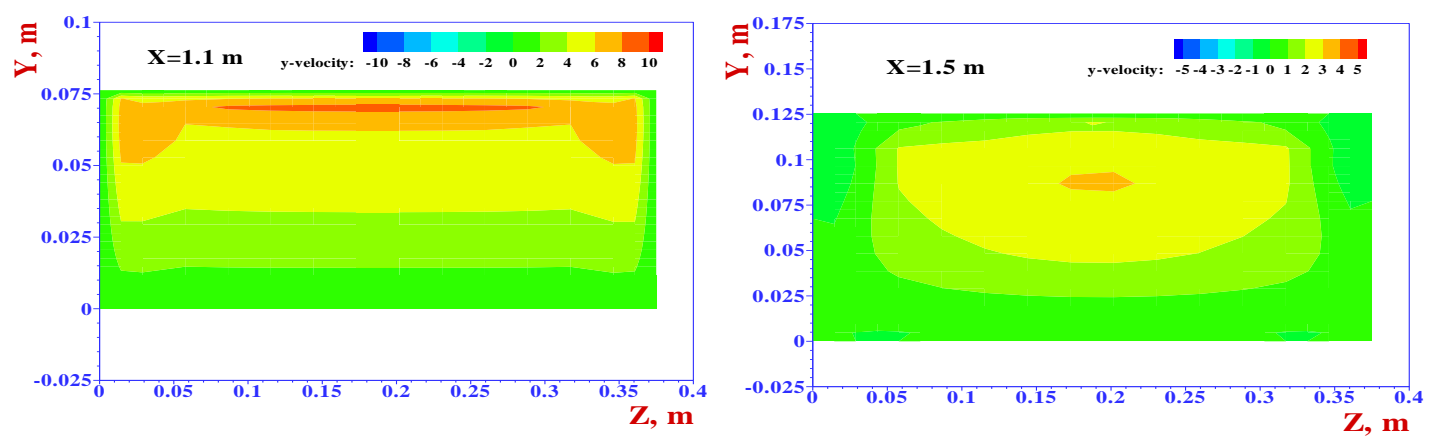

Figure $11 \mathrm{v}$ velocity contours in Z-Y plane at different $\mathrm{X}$ values.
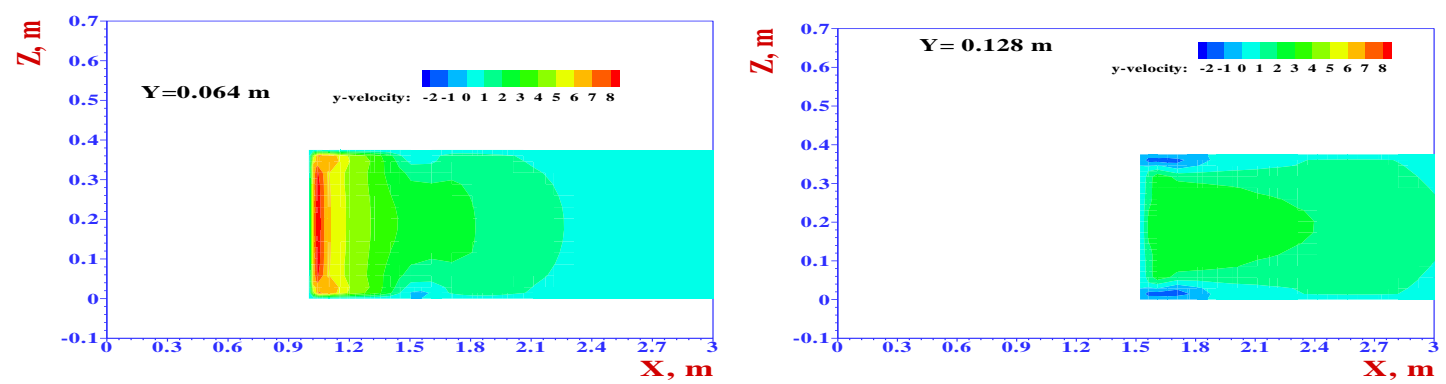

Figure $12 \mathrm{v}$ velocity contours in $\mathrm{X}-\mathrm{Z}$ plane at different $\mathrm{Y}$ values. 
To check the dimensionality of the flow, Y-velocity component (v) and Z-velocity component $(\mathrm{w})$ in many sections in the $\mathrm{X}, \mathrm{Y}$, and $\mathrm{Z}$ direction were tested.

Figs. 7-9 show the Z-velocity component contours. Fig. 7 shows the w velocity component contours through two longitudinal sections at $Z=0.037 \mathrm{~m}$ and $\mathrm{Z}=0.112 \mathrm{~m}$. Fig. 8 shows the $\mathrm{w}$ velocity component contours through $\mathrm{Y}-\mathrm{Z}$ plane (cross stream section) at two sections $X=1.1 \mathrm{~m}$ and $X=1.5 \mathrm{~m}$, and Fig. 9 shows the $\mathrm{w}$ velocity component contours through $\mathrm{X}-\mathrm{Z}$ plane at two sections $\mathrm{Y}=0.064 \mathrm{~m}$ and $\mathrm{Y}=0.128 \mathrm{~m}$. In which, the Z-velocity component distribution through the asymmetric diffuser has very small values( less than $1 \mathrm{~m} / \mathrm{s}$ ) except small regions near the boundary (separation zones). The results can show recirculating zones and non-equilibrium at $\mathrm{x} 1.1 \mathrm{~m}$ and $1.5 \mathrm{~m}$. This may be due to the adverse pressure gradient in the diffuser. From previous figures, it may be concluded that the flow in the asymmetric diffuser is almost two dimensional (w-velocity can be neglected).

Figs. 10-12 show the Y-velocity component contours. Fig. 10 shows the $\mathrm{v}$-velocity contours through the longitudinal plane at two sections $\mathrm{Z}=0.037 \mathrm{~m}$ and $\mathrm{Z}=0.187 \mathrm{~m}$. Figure 11 shows the $\mathrm{v}$-velocity contours through the span-wise plane at two sections $X=1.1 \mathrm{~m}$ and $X=1.5 \mathrm{~m}$. Fig. 12 shows the v-velocity contours through the $\mathrm{X}-\mathrm{Z}$ plane at two sections $\mathrm{Y}=0.064 \mathrm{~m}$ and $\mathrm{Y}=0.128 \mathrm{~m}$. (in which, the v-velocity has maximum value at the starting section of the diffuser and low values through the diffuser zone). It may be concluded from the figures that the change of velocity profile is very strong close to the upper wall (inclined surface) of the asymmetric diffuser.

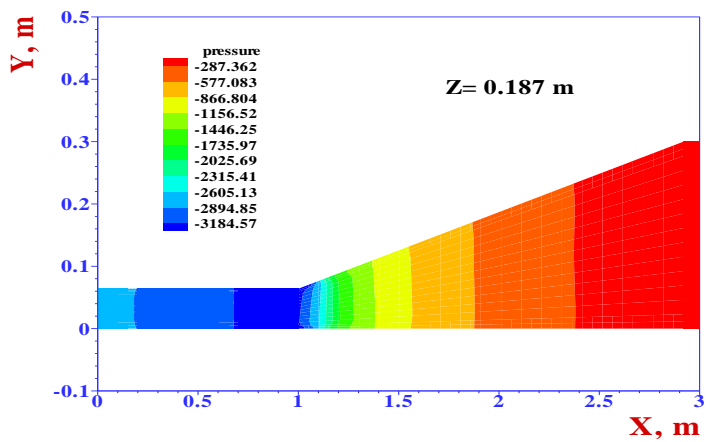

Figure 13 Pressure contours in Y-X plane at $\mathrm{Z}=0.187 \mathrm{~m}$.

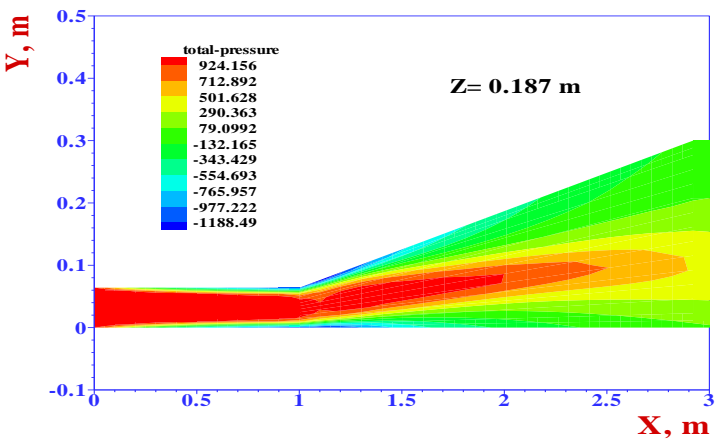

Figure 14 Total Pressure contours in Y-X plane at $\mathrm{Z}=0.187 \mathrm{~m}$.

Figure 13 shows the static pressure distribution by RNG k- $\varepsilon$ turbulence model through the longitudinal plane at $Z=0.187 \mathrm{~m}$. Total pressure distribution through the same longitudinal plane with the same turbulence model is shown in Fig. 14, in which negative pressure is found close to the boundary

\section{SUMMARY AND CONCLUSION}

9A computational and experimental study of turbulent flow through a 3-D low aspect ratio asymmetric diffuser have been presented. The flow is computed with the Fluent CFD code, using the RNG k- $\varepsilon$ turbulence model and a hexahedral grid. 
It may be concluded from the study, that although the asymmetric diffuser has low aspect ratio, the flow is almost two dimensional. Studying the Z-velocity component (w) through the computational domain may lead to a conclusion that the velocity values are small except those at the separation zones.

Both flow visualization and numerical results showed that there is a separation and non-equilibrium zone near the inclined surface (upper wall) and that there is no separation with the lower wall. . Moreover, it may be observed that the numerical model under-predicts the separation point and that, although the asymmetric diffuser has low aspect ratio; however the flow is almost two-dimensional.

\section{REFERENCES}

1. Buice, C. U. and Eaton, J. K. , "Experimental investigation of flow through an asymmetric plane diffuser," Journal of Fluids Engineering, Vol.122, pp. 433-435, 2000.

2. Mathes, A. R., "Experimental analysis of an advanced-design, quasi-radial diffuser receiving an oscillating jet, " M. Sc. Thesis, Faculty of the Virginia Polytechnic Institute and State University, p. 63, 1997.

3. Feakins, S. H., MacMartin, D. G. and Murray, R. M., "Dynamic separation control in a low-speed asymmetric diffuser with varying downstream boundary condition," AIAA 2003- 4161, pp. 8, 2003.

4. Gravemeier, V. , "Variational multiscale large eddy simulation of turbulent flow in a planar asymmetric diffuser," Center for Turbulence Research, Annual Research Briefs 2005, pp. 257-268, 2005.

5. Sbrizzai, F., Verzicco, R. and Soldati, A., "Turbulent dispersion of flyashes in a diffuser-like geometry," ICheaP-6, Sixth Italian Conference on Chemical and Process Engineering, Pisa, Italy June 8-10, 2003.

6. Törnblom, O., Herbst, A. and Johansson, A. V. , "Separation control in a plane asymmetric diffuser by means of streamwise vortices- experiment, modeling and simulation," The 5th Symposium on Smart Control of Turbulence, February 29- March 2, University of Tokyo, Japan, 2004.

7. Kaltenbach, H. J. , "Towards a near-wall model for LES of a separated diffuser flow," Center for Turbulence Research, Annual Research Briefs 1998, pp. 255-265, 1998.

8. Dahlström, S. and Davidson, L., "Hybrid RANS/LES employing Interface Condition with Turbulent Structure," Department of Thermo and Fluid Dynamics, Chalmers University of Technology, Report, Göteborg, Sweden, 2003.

9. Suh, J. , "Large eddy simulation of turbulent flow in a planer asymmetric diffuser," ME608 2002 Spring Semester Project Report, West Lafayette, Indiana, USA, 2002.

10. DalBello, T., Dippold, V., III and Georgiadis, N., J., "Computational Study of Separating Flow in a Planar Subsonic Diffuser," NASA/TM-2005-213894, 2005.

11. Iaccarino, G. , "Prediction of the turbulent flow in a diffuser with commercial CFD codes," Center for Turbulence Research, Stanford University Stanford, 
CA, Annual Research Briefs 2000, 2000.

12. Wu, X., Schlüter, J., Moin, P., Pitsch, H., Iaccarino, G. and HAM, F., "Computational study on the internal layer in a diffuser," J. Fluid Mech., Cambridge University Press, Vol. 550, pp. 391-412, 2006.

\section{دراسة عملية ورقمية لناشر غير متماثل وذو نسبة عرض إلى ارتفاع منخفضة}

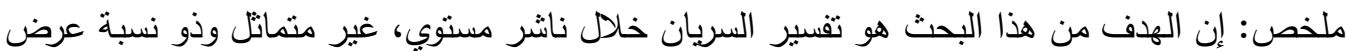

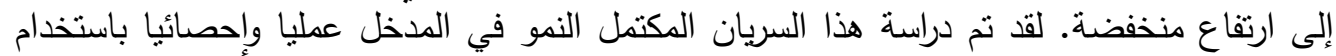

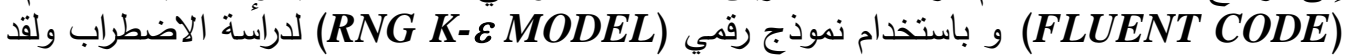

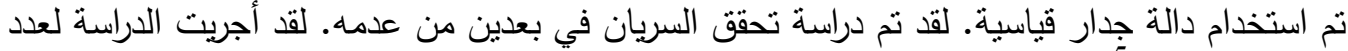
رينولاس يكافئ (3 X 10

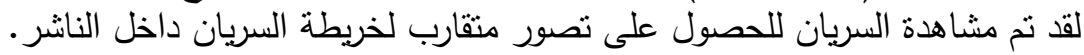

